\title{
Cytokeratin Expression and Proliferative Activity of Keratocystic Odontogenic Tumor
}

\author{
Masahide Hayakawa, ${ }^{1}$ and Hiroyuki Okada ${ }^{1,2}$ \\ Departments of ${ }^{1}$ Oral Pathology and ${ }^{2}$ Research Institute of Oral Science, Nihon University School of Dentistry at \\ Matsudo, Matsudo, Chiba 271-8587, Japan
}

Correspondence to :

Hiroyuki Okada

E-mail : okada.hiroyuki@nihon-u.

ac.jp

Keywords :

keratocystic odontogenic tumor, odontogenic keratocyst, primordial cyst, cytokeratin, proliferative activity

\begin{abstract}
Keratocystic odontogenic tumor (KCOT), called formerly as odontogenic keratocyst $(\mathrm{OKC})$, is newly classified in the odontogenic tumor by the revised WHO histological classification in 2005, because of its potential for aggressive behavior, high local recurrence rate and the involvement of PTHC gene mutation in etiology. We examined the cytokeratin expression and proliferative activity of KCOT/OKC and compared them to non-keratinized primordial cyst (PC), in order to better understand its nature. The epithelial layers of KCOT/OKC and $\mathrm{PC}$ revealed almost the same cytokeratin expression to CK 7, 13, 19, 20 and HMW, but showed the different expression to CK 17 (KCOT/ $\mathrm{OKC},+; \mathrm{PC},-)$. The LIs for $\mathrm{Ki}-67$ and topoisomerase $\mathrm{II} \alpha$ were higher in KCOT/OKC $(23.1 ; 10.4)$ than in the PC $(9.8 ; 3.6)$, and Student's $t$ test demonstrated a significant difference $(p<0.01)$ in both LIs between KCOT/OKC and PC. There was a strong correlation between the $\mathrm{Ki}^{-67} \mathrm{LI}$ and the topoisomerase II $\alpha$ LI (Pearson's correlation coefficient $r=0.91$ ), suggesting that their combined use is recommended to estimate the proliferative activity. CK 17 might be related to the proliferative lesions. It is considered that KCOT/OKC behaves biologically as a tumor and belongs a different entity to PC.
\end{abstract}

\section{Introduction}

The histopathological classification of odontogenic tumors has been newly revised by World Health Organization (WHO) and has been published by International Agency for Research on Cancer (IARC) in 2005 (1). In the re-revised WHO classification, the new term "keratocystic odontogenic tumor (KCOT)" has been used to "odontogenic keratocyst (OKC)" and the lesion has not been classified in the cyst but the tumor for the first time. OKC had been reported that some have the potential for aggressive behavior and local recurrence, and occur as multiple associated with the nevoid basal cell carcinoma syndrome (NBCCS) (2). Recent studies have disappointed the role of the human patched gene $(\mathrm{PTCH})$ in the etiology of the lesion (3-5). Based on these clinical, histopathological and experimental findings, the WHO regards the lesion as the tumor and names it keratocystic odontogenic tumor. However, as details of the histogenesis and nature of KCOT are still unclear, the classification of $\mathrm{KCOT} / \mathrm{OKC}$ is still controversial.

The purpose of the present study was to clarify the cytokeratin expression and proliferative activity of KCOT/OKC using immunohistochemistry, and to better understand its nature.

\section{Materials and Methods}

\section{Materials}

We retrieved the files on ten cases of KCOT (some were diagnosed as $\mathrm{OKC}$ ) without clinical findings of NBCCS kept at the Department of Pathology, Nihon University School of Dentistry at Matsudo. Ten cases of non-keratinizing primordial cyst (PC) were used as control. Consideration was given to patient privacy, diagnosis, and the management and progno- 
sis of the lesions.

\section{Methods}

1) Light microscopy

Following fixation with 10\% neutral formalin solution, specimens were cut into several pieces and routine paraffin sections $(4 \mu \mathrm{m})$ were prepared. The sections were stained with hematoxylin and eosin (HE).

2) Immunohistochemistry

Primary antibodies against various monoclonal cytokeratins (CKs) such as OV-TL 12/30 (CK 7), DE -K13 (CK 13), E3 (CK 17), RCK108 (CK 19), Ks20.8 (CK 20) and 35 $\beta$ E12 (high-molecular-weight (HMW) $\mathrm{CK})$ and against $\mathrm{MIB}^{-1}\left(\mathrm{Ki}^{-67)}\right.$ and $\mathrm{Ki}^{-} \mathrm{S} 1$ (topoisomerase $\mathrm{II} \alpha$ ) as the proliferative marker were purchased from a commercial source (DakoCytomation, Glostrup, Denmark). For detection of the antigens, the dextran polymer method (Envision kit, DakoCytomation) was used. Briefly, the deparaffinized sections were pretreated with microwave irradiation in citrate buffer (10 mM, pH 6.0, $5 \times 3 \mathrm{~min})$. The primary antibodies were generally used at a dilution of $1: 100$, and the incubation time was $20 \mathrm{~min}$ at room temperature, according to the instructions of the supplier. Peroxidase activity was visualized using diaminobenzidine. For evaluation of the immunostaining technique, as a negative control, mouse IgG1 (CK 7, CK 18, CK 19, HMW CK, Ki-67), IgG2a (CK 13, CK 20, topoisomerase II $\alpha$ ) and IgG2b (CK 17) were used instead of the primary antibodies during the staining procedure.

On analysis of proliferative activity, areas showing high positivity were chosen, and the average cell positive rate (LI, labeling index) was calculated after analyzing about 1,000 cells in five fields at $\mathrm{x} 400$ magnification (6). Statistical significance was determined by Student's $t$ test. Differences at $p<0.05$ or 0.01 were considered statistically significant. To evaluate the correlation between the LIs of $\mathrm{Ki}-67$ and topoisomerase II $\alpha$ in each lesion, Pearson's correlation coefficient was used.

\section{Results}

\section{Histopathological features}

The KCOT had cystic wall architecture with a lumen. The lumen wall was the parenchyma which was lined by a corrugated parakeratinized stratified squamous epithelium of about 5 to 8 cells in thickness and without rete ridges (Fig. 1). The basal layer was composed of palisaded cuboidal or columnar epithelial cells with hyperchromatic nuclei (Fig. 1). Mitotic figures were sometimes observed in the suprabasal cells. Desquamated keratin was present in the lumen of many cases. Under the epithelial parenchyma, stroma composing of fibrous connective tissue was recognized, and inflammatory change and budding of the epithelium were often seen. Daughter cyst was also observed in some cases.

The PC had cyst wall composed of non-keratinizing stratified squamous epithelium and fibrous connective tissue (Fig. 2). The structural findings were almost same as the KCOT, except for the superficial layer which showed non-keratinizing (Fig. 2).

\section{Immunohistochemistry}

1) Cytokeratin expression

In KCOT, all or most of the three (basal, spinous and cornified) layers were positive for CK 17 (Fig. 3) and 19 and HMW CK. Most of spinous and cornified layers had positive reaction to CK 13 . All of the three layers showed no reactivity for CK 7 and 20 .

PC showed almost the same reactivity except for CK 17. All of the three layers were negative for CK 17 (Fig. 4). These results of cytokeratin expression are summarized in Table 1.

\section{Proliferative activity}

Positive expression of $\mathrm{Ki}-67$ and topoisomerase II $\alpha$ was identified in all cases of KCOT and PC. Most of the positive reactions were recognized in the basal and suprabasal cells.

1) $\mathrm{Ki}-67$

The Ki-67 LIs of KCOT and PC were 23.1 and 9.8, respectively (Figs. 5, 6). Student's $t$ test demonstrated a significant difference in LI between KCOT and PC $(p<0.01)$. 

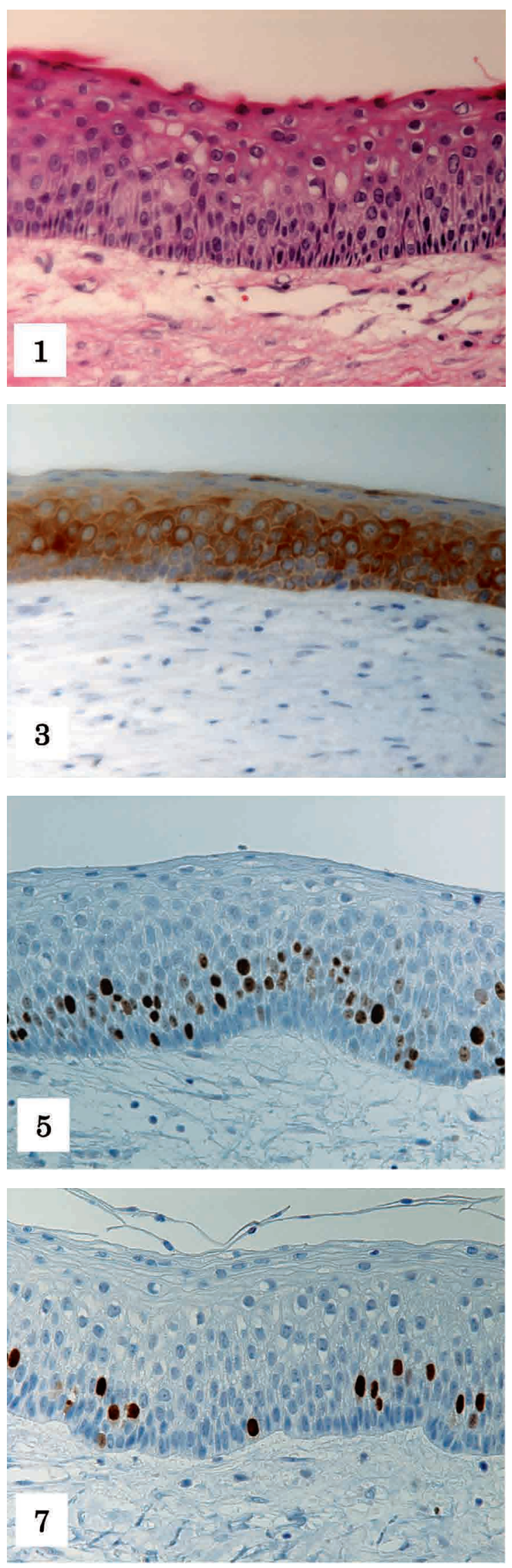
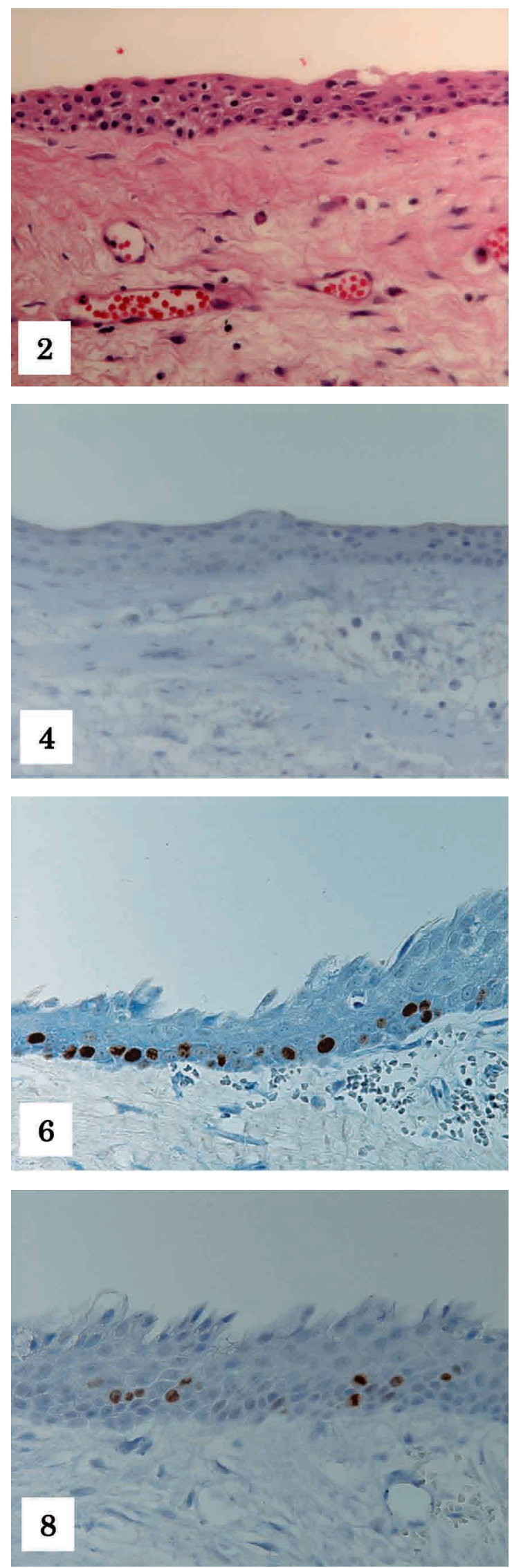
Int J Oral-Med Sci 5(1):43-49, 2006

Figs. 1, 2. Histopathological feature. The parenchyma of KCOT is lined by a corrugated parakeratinized stratified squamous epithelium, and the basal layer is composed of palisaded cuboidal or columnar epithelial cells with hyperchromatic nuclei (1). Cyst epithelium of PC is consisted with non-keratinizing stratified squamous epithelium (2). (HE, original magnification $\times 400$ ) Figs. 3, 4. Cytokeratin 17 expression. All three layers of KCOT reveal positive reaction (3), but these of PC show no positive reaction (4). (original magnification $\times 400$ )

Figs. 5, 6. Immunostaining for Ki-67. More immunopositive cells are evident in KCOT (5) than in PC (6). (original magnification $\times 400)$

Figs. 7, 8. Immunostaining for topoisomerase II $\alpha$. More positive cells are recognized in KCOT (7) than in PC (8). (original magnification $\times 400)$

Table 1. Results of immunohistochemistry

\begin{tabular}{|c|c|c|c|c|c|c|}
\hline & \multicolumn{3}{|c|}{ Keratocystic odontogenic tumor } & \multicolumn{3}{|c|}{ Primordial cyst } \\
\hline & basal layer & spinous layer & cornified layer & basal layer & spinous layer & superficial layer \\
\hline CK 7 & - & - & - & - & - & - \\
\hline CK 13 & - & $++\sim-$ & $++\sim+$ & - & +++ & +++ \\
\hline CK 17 & + & $+\sim++$ & + & - & - & - \\
\hline CK 19 & $+\sim-$ & $++\sim-$ & $+\sim-$ & $+\sim-$ & $+\sim-$ & $+\sim-$ \\
\hline CK 20 & - & - & - & - & - & - \\
\hline CK HMW & ++ & ++ & ++ & ++ & ++ & ++ \\
\hline
\end{tabular}

CK, cytokeratin; HMW, high molecular weight

many $\sim$ some cells; - , negative; + , slightly positive; ++ , moderately positive $;+++$, strongly positive

\section{2) Topoisomerase II $\alpha$}

The topoisomerase II $\alpha$ LIs of $\mathrm{t} \mathrm{KCOT}$ and PC were 10.4 and 3.6, respectively (Figs. 7, 8). Student's $t$ test demonstrated a significant difference in LI between KCOT and PC $(p<0.01)$.

The data for proliferative activity are summarized in Table 2.

3) Correlation between $\mathrm{Ki}-67$ and topoisomerase II $\alpha$

There was a strong correlation between the data for $\mathrm{Ki}^{-67}$ and topoisomerase II $\alpha$ in these lesions (Pearson's correlation coefficient $r=0.91$ ).

Table 2. Labeling index of $\mathrm{Ki}-67$ and topoisomerase II $\alpha$ $(\mathrm{Mean} \pm \mathrm{SD})$

\begin{tabular}{lcc}
\hline & KCOT & PC \\
\hline $\mathrm{Ki}^{-67 *}$ & $23.1 \pm 6.4$ & $9.8 \pm 4.5$ \\
Topoisomerase II $\alpha^{*}$ & $10.4 \pm 2.4$ & $3.6 \pm 2.1$ \\
\hline
\end{tabular}

All $p$ vales are by Student's $t$ test

*Significant difference was shown between KCOT and PC $(p<0.01)$.

\section{Discussion}

$\mathrm{OKC}$, former name of KCOT, has already been examined from clinical and histopathological viewpoints. Retrospective clinical studies showed that OKC had high recurrence rate (7-9). Histopathologically, it was reported that $\mathrm{OKC}$ frequently revealed budding of the basal layer and daughter cyst formation, and had high proliferative potential of the epithelial lining compared with other odontogenic cysts (10). Genetic studies reveal PTCH mutations have been found in $\operatorname{OKC}(4,11)$. From these findings, $\mathrm{OKC}$ is classified into odontogenic tumor as KCOT in new WHO classification of 2005. On the other hand, the concept and meaning of the term "primordial cyst" have been controversial and confusing (12). The terms of odontogenic keratocyst and primordial cyst were used synonymously. However, there is truly an intra-osseous developmental odontogenic cyst having non-keratinized squamous epithelial lining without relationship to embedded tooth. The histogenesis of KCOT/OKC and PC are still unclear. Therefore, the present study analyzed 
the difference of the cytokeratin expression and proliferative activity of the lining epithelium of keratocystic odontogenic tumor (KCOT) and nonkeratinizing primordial cyst (PC).

It has been shown that the epithelium of odontogenic cyst reveals immunoreactivity for various cytokeratins $(13,14)$. Some researchers have shown a different cytokeratin expression between OKC and other odontogenic cysts (14-16). Stoll et al (16). reported that expression of CK 17 was discernible in most of OKC cases, but only some cases of dentigerous and radicular cysts, resulting CK 17 was a valuable additional parameter to distinguish $\mathrm{OKC}$ and other cysts.

In this study, all or some layers of epithelium of KCOT/OKC revealed CK 13, 17, 19, 20 and CK HMW, but all layers showed no positivity to CK 7 and 20. These patterns of cytokeratin expression of OKC were basically same as previous studies with some difference. PC showed almost the same immunoreactivity as $\mathrm{KCOT} / \mathrm{OKC}$, suggesting they were similar odontogenic lesions. However, we demonstrated that all layers of KCOT/OKC had immunoreactivity for CK 17, but those of PC showed no reactivity. It is considered that the different cytokeratin 17 profile suggests a different histogenesis, development, or differentiation of KCOT/OKC and PC. It was reported that uterine cervical dysplasia and squamous cell carcinoma revealed CK 17 positivity $(17,18)$. Based on the data of the present study and these reports, it was considered that CK 17 might be related to the proliferative activity and malignant transformation. Therefore, CK is suggested to be a useful marker for proliferative lesions.

Proliferative activity is one of the useful indicators to know the degree of malignancy and prognosis on malignant tumor, and apply to benign lesion to reveal the character and to show whether the lesion is tumor or not (19). $\mathrm{Ki}^{-67}$, expressed in all phases of the cell cycle (G1, S, G2 and M) except for G0, is a nuclear antigen as widely used for a marker of cell proliferation. Topoisomerase II $\alpha$ shows expression mainly in the S, G2 and M phases, and has received attention as a novel proliferative marker. However, only a few reported studies have used both markers (19-21). There have been some studies of proliferative activity of $\mathrm{OKC}$ using $\mathrm{Ki}-67$, but no study has used both markers (22-25). Kichi et al (24). revealed that the Ki-67 LI was greater in OKC (18.5) of lining epithelium than in dentigerous cyst (6.7). Other studies reported that $\mathrm{Ki}-67 \mathrm{LI}$ of OKC was 10.87 (22), 16.6 (25) and 20.2 (23). The series of our department demonstrated that $\mathrm{Ki}-67 \mathrm{LI}$ and topoisomerase II $\alpha$ LI of unicystic ameloblastoma were 5.56 and 4.84, respectively (19). Furthermore, the research revealed that high LIs of these markers of calcifying cystic odontogenic tumor (former term, cyst type of calcifying odontogenic cyst; $\mathrm{Ki}-67,4.71$; topoisomerase II $\alpha, 4.32$ ) and dentinogenic ghost cell tumor (former term, solid type of calcifying odontogenic cyst ; 7.82 ; $6.56)$, that were also newly classified into odontogenic tumor (19).

In this study, the present $\mathrm{Ki}-67 \mathrm{LI}$ of $\mathrm{KCOT} / \mathrm{OKC}$ (23.1) was the highest as compared to that of the previous reports (10.87-20.2). We suggest the difference may depend upon biological heterogeneity, antibody, and technical methods etc. The $\mathrm{Ki}^{-67} \mathrm{LI}$ and topoisomerase II $\alpha$ LI of KCOT/OKC $(23.1 ; 10.4)$ were significantly higher than those of PC $(9.8 ; 3.6)$. KCOT/OKC and PC have different proliferative potential, suggesting they are different entity. It is considered that the present data reveal that KCOT/ OKC behaves biologically as a tumor and the 2005 WHO classification is appropriate. There was a strong correlation between the data for the $\mathrm{Ki}^{-} 67 \mathrm{LI}$ and the topoisomerase II $\alpha$ LI. Therefore, the combine use has an advantage to estimate the lesion from the sight of proliferative active point of view.

\section{Conclusions}

We examined several specimens of KCOT/OKC to investigate cytokeratin expression and the proliferative activity, and to reveal the histogenesis, and to compare them to those of PC, and obtained the following results :

1. The epithelial layers of KCOT/OKC revealed to CK 13, 17, 19, 20 and CK HMW, but not to CK 7 and 20. PC showed almost the same immunoreactivity, 
except for CK 17. These results indicate a different histogenesis, development, or differentiation of $\mathrm{KCOT} / \mathrm{OKC}$ and PC.

2. The LIs for $\mathrm{Ki}^{-} 67$ and topoisomerase II $\alpha$ were significantly higher in KCOT/OKC than those in the PC. These proliferative profiles suggest that KCOT/ $\mathrm{OKC}$ is truly tumor, and KCOT/OKC and PC belong different entity.

3. There was a strong correlation between the $\mathrm{Ki}$ $-67 \mathrm{LI}$ and topoisomerase II $\alpha \mathrm{LI}$, indicating that their combined use would be more appropriate to estimate the proliferative activity of the lesion.

\section{References}

1. Odontogenic Tumours. In Barnes L, Eveson JW, Reichart P, Sidransky D, editors. World Health Organization Classification of Tumours: Pathology and Genetics of Head and Neck Tumours, IARC Press: Lyon, 2005. p.283-327.

2. Shear $M$ : The aggressive nature of the odontogenic keratocyst: Is it a benign cystic neoplasm? Part 1: Clinical and early experimental evidence of aggressive behaviour. Oral Oncol, 38 : 219-226, 2002.

3. Lench NJ, High AS, Markham AF, Fume WJ, Robinson PA: Investigation of chromosome 9e22.3-q31 DNA marker loss in odontogenic keratocysts, Eur J Cancer B Oral Oncol, 32B : 202-206, 1996.

4. Barreto DC, Bale AE, De Marco L, Gomez RS: Immunolocalization of $\mathrm{PTCH}$ protein in odontogenic cysts and tumors, J Dent Res, 81 : 757-760, 2002.

5. Shear M: The Aggressive nature of the odontogenic keratocyst: Is it a benign cystic neoplasm? Part 2: Proliferation and genetic studies, Oral Oncol, $38: 323$ -331, 2002.

6. Leonardi E, Girlando S, Serio G, Mauri FA, Perrone G, Scampini S, Dalla Palma P, Barbareschi M : PCNA and Ki67 expression in breast carcinoma: Correlations with clinical and biological variables, $\mathrm{J}$ Clin Pathol, 45 : 416-419, 1992.

7. Blanas N, Freund B, Schwarts M, Furst IM : Systematic review of the treatment and prognosis of the odontogenic keratocyst, Oral Surg Oral Med Oral Pathol Oral Radiol Endod, 90 : 553-558, 2000.

8. Myoung H, Hong SP, Hong SD, Lee JI, Lim CY, Choung PH, Lee JH, Choi JY, Seo BM, Kim MJ: Odontogenic keratocyst: Review of 256 cases for recurrence and clinicopathologic parameters, Oral Surg Oral Med Oral Pathol Oral Radiol Endod, 91: 328-333, 2001.

9. Chirapathomsakul D, Sastravaha P, Jansisyanont P : A review of odontogenic keratocyst and the behavior of recurrences, Oral Surg Oral Med Oral Pathol Oral Radio Endod, 101: 5-9, 2006.

10. Li TJ, Browne RM, Matthews JB: Quantification of PCNA positive cells within odontogenic jaw epithelium, J Oral Pathol Med, 23 : 184-189, 1994.

11. Agaram NP, Collins BM, Barnes L, Lomago D, Aldeeb D, Swalsky P, Finkelstein S, Hunt JL: Molecular analysis to demonstrate that odontogenic keratocysts are neoplastic, Arch Pathol Lab Med, 128: 313-317, 2004.

12. Odontogenic cysts and tumors. In Neville BW, Damm $\mathrm{DD}$, Allen CM, Bouquot JE, editors. Oral \& Maxillofacial Pathology, WB Saunders Company: Philadelphia, 2002. p.590-610.

13. Gao Z, Mackenzie IC, Cruchley AT, Williams DM, Leigh I, Lane EB: Cytokeratin expression of the odontogenic epithelia in dental follicles and developmental cysts, J Oral Pathol Med, 18 : 63-67, 1989.

14. Meara JG, Pilch BZ, Shah SS, Cunningham MJ: Cytokeratin expression in the odontogenic keratocyst, J Oral Maxillofac Surg, 58: 862-865, 2000.

15. Shear M: The aggressive nature of the odontogenic keratocyst: Is it a benign cystic neoplasm? Part 3 : Immunocytochemistry of cytokeratin and other epithelial cell markers, Oral Oncol, 38 : 407-415, 2002.

16. Stoll C, Stollenwerk C, Riediger D, Mittermayer C, Alfer J : Cytokeratin expression patterns for distinction of odontogenic keratocysts from dentigerous and radicular cysts, J Oral Pathol Med, 34 : 558-564, 2005.

17. Smedts F, Ramaekers F, Troyanovsky S, Pruszczynski M, Link M, Lane B, Leigh I, Schijf C, Vooijs P: Keratin expression in cervical cancer, Am J Pathol, 141: 497-511, 1992.

18. Carrilho C, Alberto M, Buane L, David L: Keratins 8, 10,13 , and 17 are useful markers in the diagnosis of human cervix carcinomas, Human Pathol, 35 : 546551, 2004.

19. Tanaka T, Okada H: A comparative study of proliferative activity in neoplasm-type and cyst-type calcifying odontogenic cyst, Nihon Univ J Oral Sci, 31: 167-173, 2005.

20. Hirabayashi S: Immunohistochemical detection of DNA topoisomerase type $\mathrm{II} \alpha$ and $\mathrm{Ki}-67$ in adenoid cystic carcinoma and pleomorphic adenoma of the salivary gland, J Oral Pathol Med, 28 : 131-136, 1999.

21. Hafian H, Venteo L, Sukhanova A, Nabiev I, Lefevre B, Pluot M: Immunohistochemical study of DNA topoisomerase I, DNA topoisomerase II $\alpha$, p53, and Ki -67 in oral preneoplastic lesions and oral squamous cell carcinomas, Human Pathol, 35 : 745-751, 2004.

22. Kimi K, Kumamoto H, Ooya K, Motegi K: Analysis of apoptosis-related factors and apoptotic cells in lining epithelium of odontogenic keratocysts, Oral Med Pathol, 5 : 35-40, 2000. 
23. Nakamura N, Mitsuyasu T, Mitsuyasu Y, Taketomi T, Higuchi Y, Ohishi M: Marsupialization for odontogenic keratocysts: Long-term follow-up analysis of the effects and changes in growth characteristics, Oral Surg Oral Med Oral Pathol Oral Radiol Endod, 94 : 543-553, 2002.

24. Kichi E, Enokiya Y, Muramatsu T, Hashimoto S, Inoue $\mathrm{T}$, Abiko $\mathrm{Y}$, Shimono $\mathrm{M}$ : Cell proliferation, apoptosis and apoptosis-related factors in odontogenic keratocysts and dentigerous cysts, J Oral Pathol Med, $34:$ 280-286, 2005.

25. Kolář Z, Geierová M, Bouchal J, Pazdera J, Zbořil V, Tvrdý P: Immunohistochemical analysis of the biological potential of odontogenic keratocysts, J Oral Pathol Med, 35 : 75-80, 2006. 